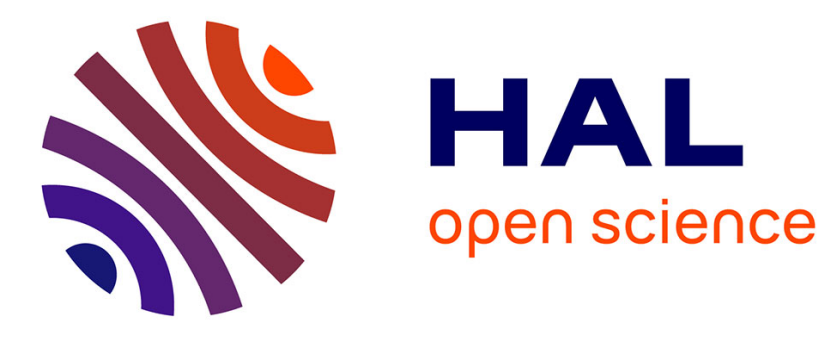

\title{
Specific tools for studying the optical response of heterogeneous thin film layers
}

Judikaël Le Rouzo, David Duché, Carmen M Ruiz, François Thierry, Miriam Carlberg, Gérard Berginc, Marcel Pasquinelli, Jean Jacques Simon, Ludovic Escoubas, François Flory

\section{To cite this version:}

Judikaël Le Rouzo, David Duché, Carmen M Ruiz, François Thierry, Miriam Carlberg, et al.. Specific tools for studying the optical response of heterogeneous thin film layers. Journal of Nanophotonics, 2017, 11 (1), 10.1117/1.JNP.11.016009 . hal-01788933

\section{HAL Id: hal-01788933 \\ https://hal-amu.archives-ouvertes.fr/hal-01788933}

Submitted on 9 May 2018

HAL is a multi-disciplinary open access archive for the deposit and dissemination of scientific research documents, whether they are published or not. The documents may come from teaching and research institutions in France or abroad, or from public or private research centers.
L'archive ouverte pluridisciplinaire HAL, est destinée au dépôt et à la diffusion de documents scientifiques de niveau recherche, publiés ou non, émanant des établissements d'enseignement et de recherche français ou étrangers, des laboratoires publics ou privés. 


\title{
Specific tools for studying the optical response of heterogeneous thin film layers
}

\author{
J. Le Rouzo ${ }^{a}$, D. Duchéa ${ }^{a}$ C. M. Ruiz ${ }^{a}$, F. Thierry ${ }^{a}$, M. Carlberg ${ }^{a}$, G. Berginc ${ }^{b}$, M. Pasquinelli ${ }^{a}$, \\ J-J. Simon ${ }^{\text {a }}$, L. Escoubas ${ }^{\text {a }}$, F. Flory ${ }^{\text {a c }}$ \\ ${ }^{a}$ Aix Marseille Université, CNRS, Université de Toulon, IM2NP UMR 7334, 13397, Marseille, France; \\ ${ }^{\mathrm{b}}$ Thales Optronics, 2 avenue Gay Lussac, 78990, Elancourt, France ; ${ }^{c}$ Ecole Centrale Marseille, 13451 Marseille, France
}

\begin{abstract}
The extraordinary progresses in the design and realization of structures in inorganic or organic thin films, whether or not including nanoparticles, make it possible to develop devices with very specific properties. Mastering the links between the macroscopic optical properties and the opto-geometrical parameters of these heterogeneous layers is thus a crucial issue. We propose to present the tools used to characterize and to model thin film layers, from an optical point of view, highlighting the interest of coupling both experimental and simulation studies for improving our knowledge on the optical response of the structure. Different examples of studies are presented on CIGS, Perovskite,P3HT:ZnO, PC70BM, organic layer containing metallic nanoparticles and colored solar cells.
\end{abstract}

Keywords: Light management, thin films, optical properties, photonics

E-mail : judikael.le-rouzo@univ-amu.fr

\section{INTRODUCTION}

The complexity of the interaction between light and heterogeneous matter highlights the importance of knowing the optical and electronical properties of the material, in order to be able to design the optical responses [1]. We would like to be able to do this, however it is not so easy. The understanding of the links between the macroscopic optical properties (refractive index and extinction coefficient), opto-geometrical parameters of the heterogeneous layers and their optical responses concerns various applications in the optical range of wavelengths (control of the emissivity of the surfaces, modeling of active imaging techniques, the realization of new types of absorbers in thin film form working in the visible and infrared bands, refractive index gradient nano-films). Areas of interest are numerous such as thermal solar and photovoltaic (selective absorbers, anti-reflective, rough transparent conductive oxides, etc...) and more generally optoelectronics (special optical coatings, more sensitive photodetectors, etc...). And the possibility of realizing thin films in the form of complex structure, comprising for example nanoparticles, or offering photonic patterns, allow to design new optical behavior of a material.

The investigations for mastering the light behavior in a layer not only led researchers to use different photonic concepts but also to look for new materials with specific properties such as the mechanical flexibility, the low specific weight, the color or transparency.

The architecture of thin films can be relatively complex and requires a thorough understanding of the materials' behavior, involving accurate knowledge of the electrical, optical, and structural properties of the materials that compose the layers. The use of both experimental and simulation optical tools are then required in order to improve the optical design of the thin-film. This approach of coupling characterization and modeling provides a correct prediction and validation of device performance. This work, which is directly related to the fields of photonics, also involves knowledge in other parts of the Physics (e.g. Solid State, Electronic).

In this paper, we will first describe briefly the phenomena of light-matter interaction which can occurs in thin films. Then, we will present different devices which are under study in our laboratory, i.e. thin film solar cells, and heterogeneous thin films made of nanoparticles embedded in a polymer host matrix. After giving some details about both these structures and their basics properties we will focus our attention to the means used to measure and to model the optical properties of these thin films. The optical properties of each material are of great importance on the final performance of the device, and are based on a comprehensive set of very precise measurements. However, this is not enough, and models are needed to define the final optical parameters of new materials. 


\section{LIGHT-MATTER INTERACTION}

\subsection{Materials, Thin film layers}

First, we mention that in this paper, we focus our study to wavelengths between $200 \mathrm{~nm}$ and $5 \mu \mathrm{m}$, which is the range of the electromagnetic spectrum of interest. We consider either metallic, semiconductor or even dielectric material in thin film form, at micrometric or nanometric scale.

The interaction between light and matter is strongly linked to both the structure of the matter, and of the light wavelength. The composition of the matter greatly influences the propagation of electromagnetic waves, and especially the optical constants (refractive index $\mathrm{n}$ and extinction coefficient $\mathrm{k}$ ). When an electromagnetic radiation reaches the surface of a layer, different physical mechanisms are involved (see figure 1). A portion of the light may optionally pass through the medium if it is sufficiently transparent. Another part will be reflected back in the incident medium, and finally a part of the light may propagate within the material. In this latter case the light may be either scattered or absorbed, depending on both the structure and the optical properties of the material. Typically, a conductive medium (metal) exhibits absorption and also leads to a strong reflection. A dielectric medium on the other hand will, in its transparency region, allow the propagation of the light wave over long distances. And in the case of a semiconductor, depending on its energy bandgap, the light will be absorbed or not. We also have to mention the possibility of emission of photons, phenomena of luminescence of matter due to radiative relaxation.

We also have to point out that the ratio between the wavelength and the dimension of the layer influences the Physics used for explaining the optical waves behavior [2]. In optics, we are use to compare the critical dimension with the diffraction limit in free space propagation, i.e. when it is smaller than the wavelength of light. Indeed, in the case of quantum structures with typically sizes of a few nanometers, classical physics is in default. In this paper, we still consider in most cases material dimension close to the wavelength or smaller than $\lambda / 10$. For the study of nanoparticles, we will use the effective medium approximation.

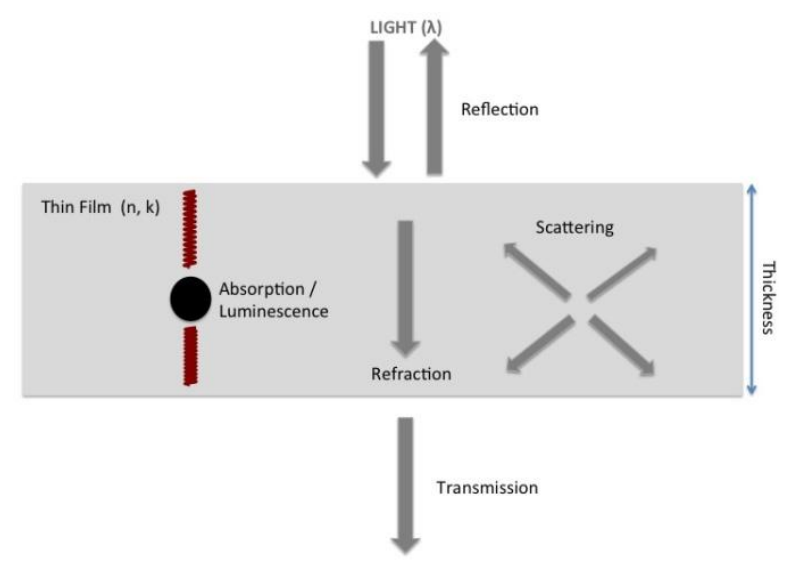

Figure 1. Optical phenomena which may occur in an optical thin film with optical constants $\mathrm{n}$ and $\mathrm{k}$.

\subsection{Photonic structures, nanoparticles and light management}

Several approaches or new structures can be used in optoelectronic devices in order to improve their performances. Different routes are possible: the first is to minimize reflection at the air-material interface with anti-reflective coatings such as thin film layers or structured surfaces (see figure 2, [3]). The second is based on the engineering of the electromagnetic field to control its distribution in the thickness of a stack of thin films [4]. To go further, we can appeal to more innovative optical concepts, based on photonic crystals, which are structures whose refractive index varies periodically in one or more dimensions of space in order to control and modify the propagation characteristics of a light 
wave. Photonic crystals are used to increase light absorption in thin film solar cells [5]. Another alternative to increase the absorption of the photons in an active layer is to use plasma resonance of electrons at a metal / dielectric interface, which can be excited by the incident light beam. This field is known as plasmonics. The inclusion of metal nanoparticles in a layer allows to couple localized plasmons resonances and thus to change the optical response of the thin film [6].

In this context, new concepts or new architectures for solar cells are more and more in use nowadays, due to the aim of the photovoltaic technology which promotes good efficiency at low-cost. Associated to low cost fabrication process thin film solar cells are good candidates to respond to this objective. However, to reach good efficiency we need to use together different photonic concepts.

We will show that the control of the light inside the cell is of great interest for the development of $3^{\text {rd }}$ generation solar cells made of thin film layers. And not only for the efficiency but also to design a new kind of solar cells like "Transparent and colored solar cells", interesting for the Building Integrating PhotoVoltaic (BIPV). In thin film solar cells, the apparent color and transparency is given by its reflected and transmitted spectra. We discuss on light management such as the use of Bragg mirrors, of diffraction gratings, of surface plasmonic effect or of photonic crystal to optimize the cell performances while controlling their reflected and transmitted spectra and by taking into account the human eye sensitivity.

We also work on organic thin films containing metallic nanoparticles which are interesting for different applications, including thin film solar cells [7]. Our aim in this study is to precisely define the influence of these nano-objects, embedded in an organic matrix, on the optical response of a thin film.

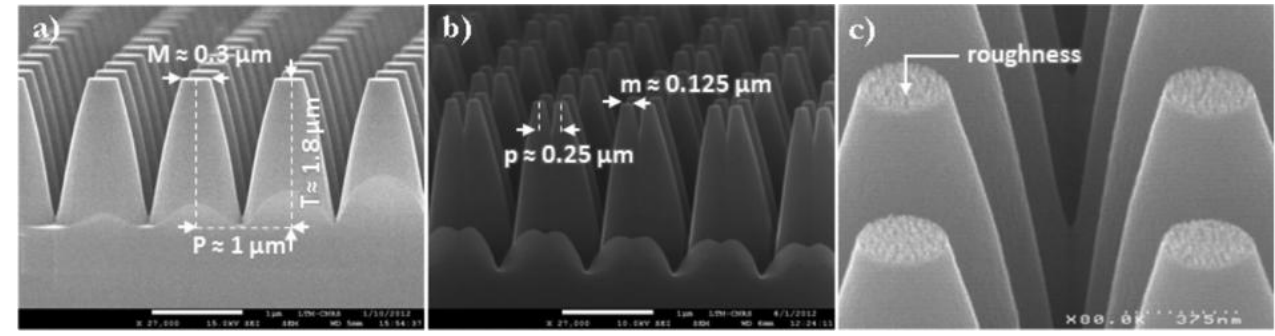

Figure 2. SEM pictures of antireflective pyramidal structures made of silicon, working in the visible and infrared spectral range. a) flat-topped b) patterned top c) rough-topped silicon cones grating. Images extracted from reference [3].

\section{THIN FILM LAYERS}

\subsection{Thin film solar cells}

Nowadays, it exists various photovoltaic solar cell technologies, which are usually sorted in three generations [8]. The first one is based on silicon material and relies on the PN junction made with this semiconductor. The maturity of this technology and the good module efficiency (over 20\%) make the silicon PV market leader (90\%). However, PV specifications involve thinking about other technologies. Indeed, the reduction of the raw material, improving performance, adaptation to a specific market (flexible, color...), are parameters that are difficult to reach with silicon technology.

The second and the third generation of photovoltaic try to overcome certain limitations of the first generation. Among all the technologies of these generations we focus our work on thin film solar cells, using inorganic or organic semiconductor materials with high absorption coefficients. The inorganic materials used are named CIGS, CZTS, perovskite.. CIGS, and CZTS photovoltaic devices are polycrystalline thin films. These cells consist of stacks of thin layers of different materials. Depending on the stoichiometry of the chalcopyrite, the bandgap ranges from 1.04 and 1.68 $\mathrm{eV}$ for CIGS solar cells and from 1.0 to $1.5 \mathrm{eV}$ for kesterite solar cells. These values are in the range of energy that allows high solar-energy conversion [9].

The organic semiconductor materials present significant opportunities for the production of organic solar cells (OSCs) for various reasons: their easy manufacturing process enables large scale production at low cost (roll-to-roll and ink jet 
techniques); they are easy to integrate (flexible substrate); the organic aspect offers a variety of structures (polymers, small molecules) and functionality (separate adjustment of the bandgap ...). If the organic materials have the advantage of having strong absorption coefficients (more than $10^{5} \mathrm{~cm}^{-1}$ ), the low mobility of the charge carriers $\left(10^{-6}\right.$ to $100 \mathrm{~cm}^{2} . \mathrm{V}^{-}$ ${ }^{1} . \mathrm{s}^{-1}$ ) limits the thicknesses of the films to $100-200 \mathrm{~nm}$, that is to say at much lower values than 400 or 500 nanometers required to absorb a major portion of the solar spectrum. It is therefore necessary to increase the interaction between the incident light and the photoactive layer. Moreover, the exciton diffusion lengths do not exceed $10 \mathrm{or} 20 \mathrm{~nm}$ in the majority of organic materials; so this is the maximum distance that can be tolerated between two donor-acceptor material interfaces. In summary, all these dimensional constraints require that the optimal structure of the active layer of an organic solar cell has to be an interpenetrated structure type (named bulk heterojunction (BHJ)) [10].

In the last years, the perovskite solar cell technology has attracted a lot of attention. The structure of the organometal halide perovskites is of $\mathrm{ABX} 3(\mathrm{~A}=$ alkyl-NH3, $\mathrm{B}=\mathrm{Pb}$ or $\mathrm{Sn}, \mathrm{X}=\mathrm{I}, \mathrm{Cl}$, or $\mathrm{Br}$ ). Due to these special properties, such as very strong absorption and a tunable bandgap (from $1.57 \mathrm{eV}$ up to $2.3 \mathrm{eV}$ covering the visible spectrum up to the nearinfrared) by modifying the chemical composition (e.g., alloying iodine with bromine for the X-site composition), this hybrid material is very promising for the field of photovoltaics [11].

The figure 3 presents the typical structure of the thin film solar cells described above and which are under study in this work. We have to notice that the whole thickness of each stack is ranging from hundred nanometers (organic and perovskite) to a few micrometers (CIGS and CZTS). All of these cells have also the possibility to be made on flexible substrate.

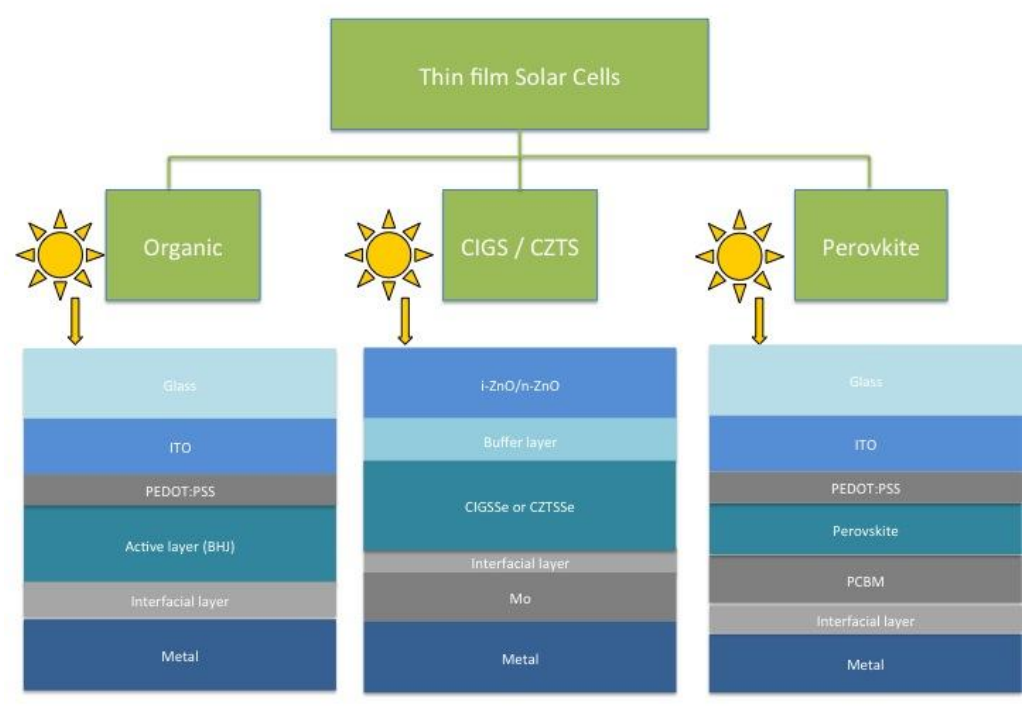

Figure 3. Typical structures of thin film solar cells. From left to right, organic solar cell, CIGS/CZTS solar cells and Perovskite solar cell.

\subsection{Organic thin films with nanoparticles}

The rapid progress in nanotechnology in the last years allowed the realization of well controlled nanostructures with specific properties more varied than those of bulk materials. Structured materials at the nanometer scale are used in optoelectronic applications because their optical and electrical properties can be controled by changing their nature, composition or their geometry. At such a scale of a few nanometers the electron confinement effects change with the spatial dimensions. Technological applications based on nanostructures are numerous. The most affected areas are optoand nanoelectronics, biotechnology and aerospace for which the mechanical properties of nanocomposites are interesting. Here, we present some work done on metallic nanoparticles for optoelectronic applications. These nanostructures are enclosed in a polymer host matrix. The nanoparticles in polymer thin films are assumed to result in layers with tunable optical properties. 
Silver nanoparticles of different shapes and sizes are chemically synthesized to be deposited in thin layers with a view to obtain transparent layers which absorb light over a wide spectral range. The nanoparticles are deposited in thin layers into the polymer, soluble in water, non-absorbing in the visible and compatible with silver nanoparticles (see figure [4]).

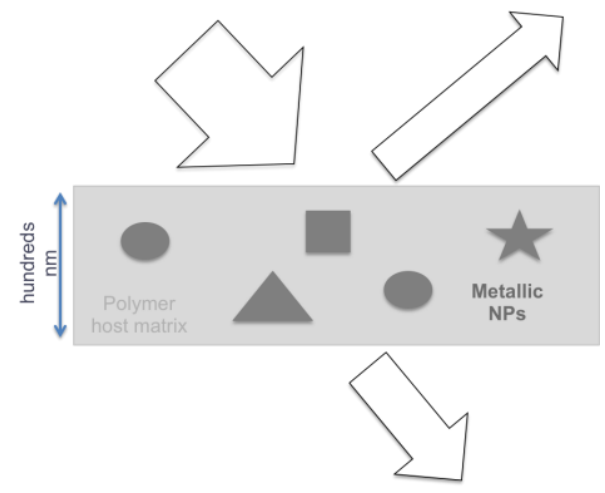

Figure 4. Heterogeneous thin film layer under study.

\section{SET OF OPTICAL MEASUREMENTS}

\subsection{Objectives}

In this section, we briefly describe the different optical characterization techniques which can be used together in order to define entirely the optical properties of thin film layers. If we want to develop accurate models to confirm or to predict our measurements, we need to know the basic optical parameters such as the optical index or the dielectric permittivity. These parameters can be linked to the absorption processes, and as we know from thin-film solar cells, they can vary strongly with the deposition method. Also, the range of optical response of the material (reflection, absorption, transmission) will be an important parameter for the design of the layer. All of these values can be obtained with a combination of spectroscopic ellipsometry (SE) measurements of the optical indices and spectrophotometric measurements of the R, T, and A (reflectance, transmittance, and absorption) spectra.

Obviously, to be quite precise, a complete study of a thin film layer should include at least structural techniques (e.g., electronic microscopy, x-ray scattering, ultraviolet photoelectron spectroscopy, and atomic force microscopy) and for thin film solar cells, electrical techniques (e.g., conductance measurements, Hall effect, and deep-level transient spectroscopy), which also contribute to improve the elaboration of the layers. For instance, a conductive-Atomic Force Microscopy with possibility of realizing Kelvin Probe-AFM measurements in dark and under light bias of different wavelengths could help to understand the impact of the structure on both optical and electrical behavior at the microscopic level.

In this paper we focus our attention on optical measurements but we should keep in mind to consider electrical measurements to have a better understanding of the whole behavior of a material or a device.

\subsection{Ellipsometry}

The spectroscopic ellipsometry (SE) is an optical technique used for surface and thin film analysis. SE is a very efficient tool to determine the optical indices of bulk materials and materials in thin-film form ( $\mathrm{n}$ and $\mathrm{k}$ ). The ellipsometric measurements consist in measuring the change in polarization after reflection on a flat surface, and what we get are the two ellipsometric parameters $(\tan \Psi$ and $\cos \Delta)$. These latter are thus extracted from the ratio between the Fresnel coefficients in p-polarization (rp) and in s-polarization (rs), which can be formulated as follows:

$$
\frac{r_{p}}{r_{p}}=\tan e
$$


where $\tan (\Psi)=|\mathrm{rp}| /|\mathrm{rs}|$ represents the relative amplitude attenuation and $\Delta=\arg (\mathrm{rp})-\arg (\mathrm{rs})$ is the phase shift between the p- and s-polarizations. A dispersion model is then elaborated to determine the dielectric constant of the materials $(\varepsilon=$ $\varepsilon 1+i^{*} \varepsilon 2$ ). The absorption (A) of the layer is thus predicted thanks to a transfer matrix method (TMM) and using optical indices ( $\mathrm{n}$ and $\mathrm{k}$ ) extracted from variable angles SE. Finally A is compared to the A deduced from reflectance (R) and transmittance $(\mathrm{T})$ measurements. For thin films of new materials, or thin films with nanoparticles, this study is very challenging because of the anisotropy of the layer and/or the surface roughness. To be sure of getting the correct indices, it is possible to use a two-layer model with a surface roughness correction using a Bruggeman effective medium approximation (EMA).

In addition, such method is generally limited by the accurate calibration of the optical instruments. This could lead to important discrepancies between the measured absorption spectra and the predicted ones from the ellipsometric results assuming an isotropic model. The optical indices can thus serve as input parameters to model the optical performances of thin-film, but they are also of high interest for the extraction of information on the electronic properties of the materials . Indeed SE is of high interest not only for nondestructive measurements of thicknesses and optical indices but also for nondestructive measurements of the electronic transitions in materials within thin-film stacks [12].

\subsection{Photoluminescence and Photoreflectance}

Photoluminescence (PL) and/or photoreflectance (PR) measurements are useful for studying the electronic levels inside the layers. More, the combination of photoluminescence technique with photoreflectance technique can be employed for the analysis of the different layers of ultrathin devices and, in particular, the simultaneous determination of their fundamental transitions and related default.

Photoluminescence spectroscopy provides information about the structure of each layer, e.g. analysis of optical transitions inside the band of the material. PL mapping also brings light into possible inhomogeneity related with the advanced structure of the devices. PL measurements can be performed at different temperature, especially at low temperature, and with different excitation wavelengths for the analysis of optical confinements and other optical phenomena related to the structure of the thin films. The PL technique investigates the emission of light from a solid due to the deviation of the system from the thermal equilibrium. It particularly relates to the deviation of the equilibrium via the absorption of light with energy higher than the bandgap of the material. The luminescence is due to both the relaxation of electrons to lower energy states and the simultaneous emission of photons. We have to notice that not all the possible relaxation processes will generate photons, since the transition should be dipole-allowed for a successful photon emission to occur. Among them, there is a set of different transitions that are possible to see in a semiconductor used in photovoltaics (these transitions are presented in Fig. 5) [13].

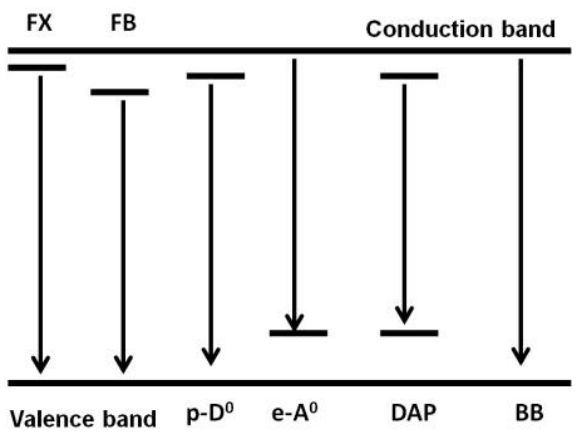

Figure 5. Schematic representation of the different origins of transitions that can be observed by photoluminescence measurements. From left to right the free (FX) and bound (FB) excitonic transitions, the free-bound transitions (p-D0 and e-A0), the Donor-Acceptor Pair and the Bound to Bound transitions) [14].

For obtaining the bandgap of any semiconductor, the most precise technique is the photoreflectance. With this technique we can also see interband transitions in the range of measurements. Indeed, from photoreflectance measurements it is possible to analyze the band configuration at the interface, by measuring the fundamental optical transitions. As the band structure of any semiconductor is very sensitive to modifications such as diffusion or structural strains, this technique permits to detect possible interplaying of the cell structure with the basic properties of an absorber and thus, the performance of a solar cell. While PL is a technique used to study the interaction between the semiconductor and light, and needs a minimal amount of material to provide an interpretable signal, reflectance-based techniques exploit the 
sudden variation of the optical indices at an interface. This means that they are much more sensitive to small quantities of material and can bring information on what is really happening at the interface between two different materials. PR belongs to the family of modulated reflectance techniques such as electroreflectance or piezoreflectance. The main advantage of PR is that, as it uses an optical modulation, the whole setup is optics based, and does not require any previous preparation of the sample for analysis such as contact deposition $[15,16]$.

\section{OPTICAL STUDY OF HETEROGENEOUS THIN FILMS}

In this last section, we propose to show some examples of optical studies made on our samples, which have been presented in section 3. Our objective is to highlight the accuracy of our measurements and the link with at first the optical behavior of the material but also with the structural properties of some of them and with the performances of the device. These results are not exhaustive, and the choice that we did for this paper could be extended to a large panel of other structures.

\subsection{Photoluminescence and Photoreflectance of thin film solar cells}

As discussed in section 3, the bandgap of the perovskite material is tunable between 1,57 eV and 2,3 eV, depending on the stoichiometry of the layer, which is of great interest for multijunctions solar cells. Regardless of the deposition method of the perovskite, we must validate the energy gap depending on the composition of the layer, including the ratio I / Br. The combination between photoluminescence and photoreflectance measurements, is a very powerful tool by providing a very accurate measurement of the fundamental optical band gap of the semiconductor.

The figure 6 presents a study both by PL and PR of organo-metallic perovskites, $\mathrm{CH} 3 \mathrm{NH} 3 \mathrm{PbCl}_{\mathrm{x}}\left(\mathrm{I}_{\mathrm{y}} \mathrm{Br}_{1-\mathrm{y}}\right)_{3-\mathrm{x}}, \quad$ with different $\mathrm{I} / \mathrm{Br}$ ratios. The dotted lines of the same color as the PL peak indicate the band gap position calculated from PR spectra. On the right, a summary table with the PL peak positions, the PR bandgaps calculated from measurements and the $\mathrm{k}$ values from the PL power dependence study for all the compositions [17].

While the PL peaks are all red shifted and very near to the pure iodine peak, the PR bandgap position follow the expected distribution. The values in the table, show the differences between the PL maximum position and the PR bandgap. The reason for the shifting of the PL spectra is not yet clear. A discussion must be held on the PL measurements and on the transitions, which occur in these samples. Some Raman spectra measurements could help us on this study and for the simultaneous analysis of their fundamental and defect related transitions.

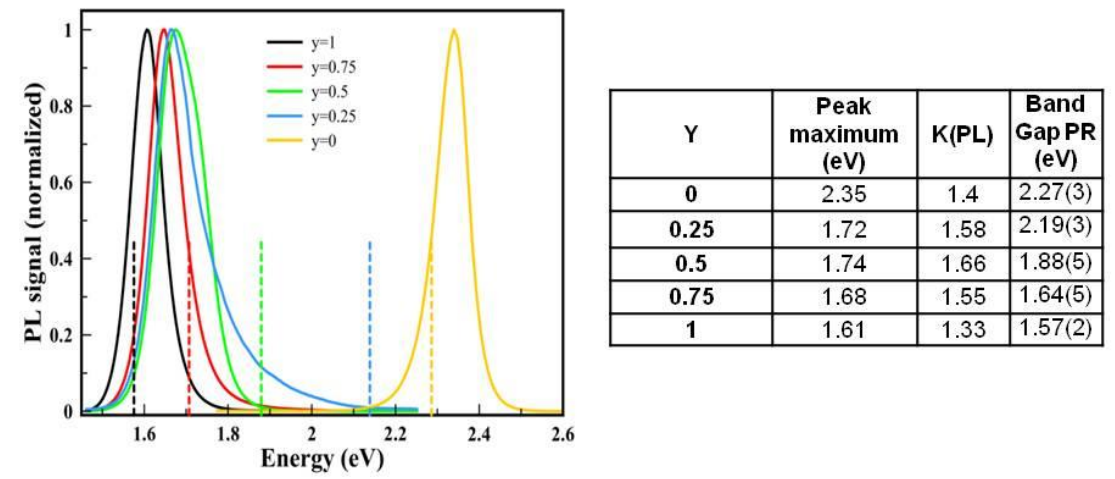

Figure 6. PL peaks for different $\mathrm{I} / \mathrm{Br}$ ratios in $\mathrm{CH} 3 \mathrm{NH} 3 \mathrm{PbCl}_{\mathrm{x}}\left(\mathrm{I}_{\mathrm{y}} \mathrm{Br}_{1-\mathrm{y}}\right)_{3-\mathrm{x}}[14]$ and $\mathrm{PR}$ results [17].

A PR study on CIS solar cells is presented in the figure 7. These PR measurements can be performed to analyze the origin of the current density $\left(\mathrm{J}_{\mathrm{SC}}\right)$ deviations in a set of $\mathrm{CuInS} 2 / \mathrm{CdS}$ solar cells fabricated in the same nominal conditions, and can be compared with structural and electrical characterizations [18]. When PR spectra are obtained for a low and a high $\mathrm{J}_{\mathrm{SC}}$ device, the resulting transitions are different, as it can be seen in Figure 6. By studying also the Raman scattering of these layers we observe that small fractions of the CIS $\mathrm{Cu}-\mathrm{Au}$ phase (CA) appears for the samples 
with better $\mathrm{J}_{\mathrm{SC}}$, leading to the conclusion that having small amounts of this defective phase helps to minimize the strain in the chalcopyrite phase CIS $(\mathrm{CH})$, improving their transport properties.
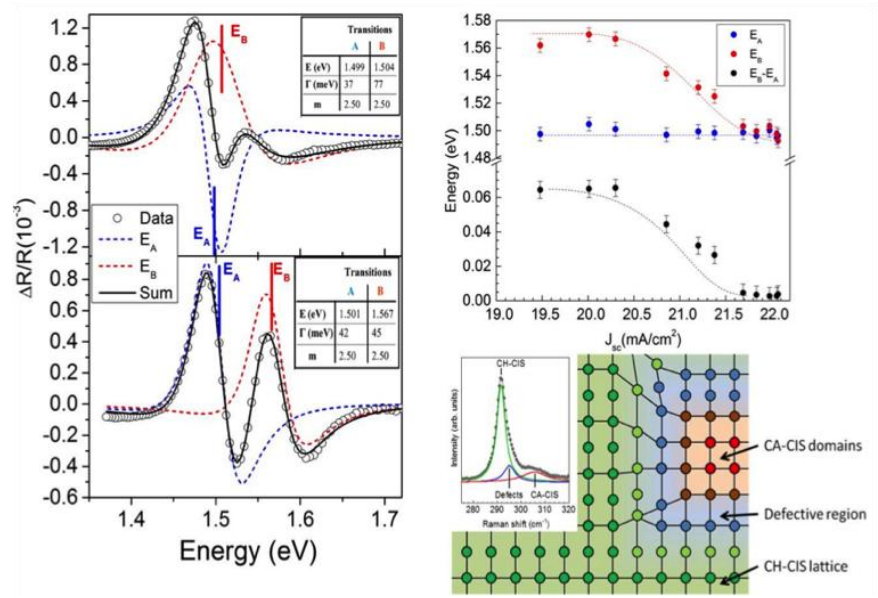

Figure 7. PR spectra of a high Jsc and low Jsc CIS solar cell, showing the splitting of the valence band for the low Jsc values (right, top) that originates from strain in the material related with the presence of $\mathrm{Cu}$-Au regions. [18]

\subsection{Ellipsometric study of thin film}

Here, we present different ellipsometric studies on different thin films. As presented before, this technique is an interesting tool to determine the thickness and the optical indices ( $\mathrm{n}$ and $\mathrm{k}$ ) of a layer. However, although the measurement is not so complicated, the model that is used for fitting the values can become quickly difficult in the case of particular materials or surfaces (rough or anisotropic).

The first example concerns a hybrid layer made of a blend between an inorganic semiconductor material and an organic material. In our case, we work on a bulk heterojunction in $\mathrm{ZnO}$ and P3HT (poly(3-hexylthiophene-2,5-diyl)) (ratio 2:1), which propose bandgaps within the spectral range of the ellipsometer. The optical indices $\mathrm{n}$ and $\mathrm{k}$, presented on top of the figure 8, are obtained using Tauc-Lorentz models [19]. The values of the correlation coefficients of these fits indicate appropriate fits to the data. On this figure, the reflection $[\mathrm{R}(\lambda)]$ and the transmission $[\mathrm{T}(\lambda)]$ spectra for P3HT:ZnO (1:2) is also represented (on bottom of the figure). A comparison is made between these measurements and the optical simulations done by TMM using the optical indices presented before and the film thicknesse measured with a mechanical profilometer, as input parameters. We can see a good agreement between the calculated and the measured spectra which demonstrates the reliability of these optical indices $n$ and $\mathrm{k}$ to predict the real optical properties of the films. 

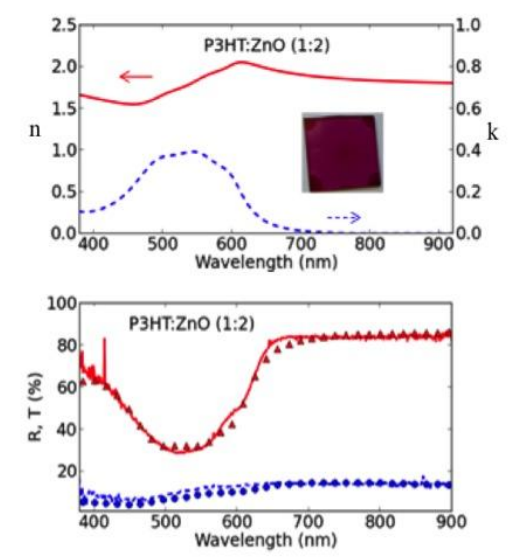

Figure 8. Top : Optical indices $\mathrm{n}$ (continuous lines) and $\mathrm{k}$ (dotted lines) as a function of the wavelength $\lambda$ for P3HT:ZnO (1:2) (Inset is a picture of the sample.) Down : Comparisons between experimental measurements of T (-) and R (. . ) and optical simulations of $\mathrm{T}(\Delta)$ and $\mathrm{R}(\mathrm{O})$ for layers coated on glass substrates [20].

Recently, a study has shown that a sum of Gaussian oscillators can be used to model the imaginary part of the dielectric function of organic semiconductors such as PC70BM ([6,6]-phenyl-C71-butyric acid methyl ester) [12]. In ellipsometric study, Gaussian oscillators set from absorbance measurements, are used for the dispersion model elaboration, and specifically to model the absorption line shape for organic semiconductors. Thanks to its suitability to describe strong electron-phonon coupling in $\pi$-conjugated molecules, electronic transitions can be extracted from ellipsometric measurements for PC70BM in the UV-visible range. The figure 8 shows the imaginary part of the dielectric constant of PC70BM, and some electronic transitions. We found a very good agreement between electronic transition values obtained from SE measurements and literature data. It demonstrates the reliability of the dispersion model and shows the potential of SE measurements for the determination of the optoelectronic properties of materials.

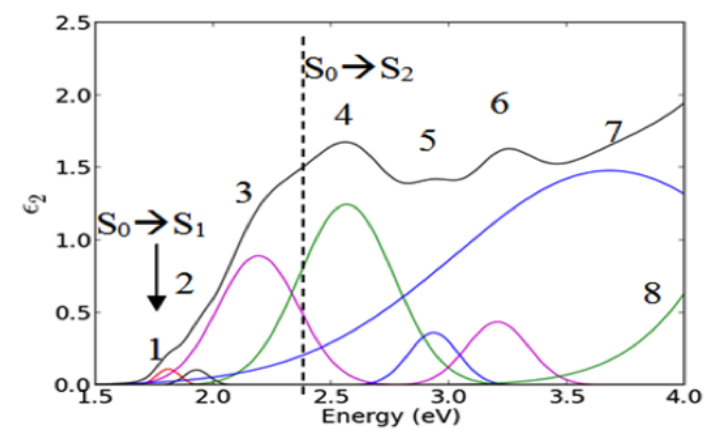

Figure 9. Imaginary part of the PC70BM organic material and Gaussian oscillators which have been used for the dispersion model elaboration [12].

The last example we present in this section, is related to an optical study performed on a thin film of metallic nanoparticles embedded in PVP polymer (thickness of hundred nanometers). The nanoparticles are in the shape of nanoprisms. The ellipsometric measurements on this kind of heterogeneous layer are not so easy, due to the dispersion of nanoparticles inside the polymer and also due to a possible roughness of the layer surface. Both the refractive index and the extinction coefficient are shown on the figure 10 (on the left). The k coefficient presents a large peak at $700 \mathrm{~nm}$ with a broadening of $150 \mathrm{~nm}$ at width-half, which corresponds to the plasmonic resonance of the nanoprisms inside the polymer matrix. The TMM method is used to calculate the reflection of the layer from ellipsometric results. The figure 10 , at the right, showing the comparison between this calculation and our spectrophotometric measurement, allows us to be more confident on the optical indices found with our dispersion model. 

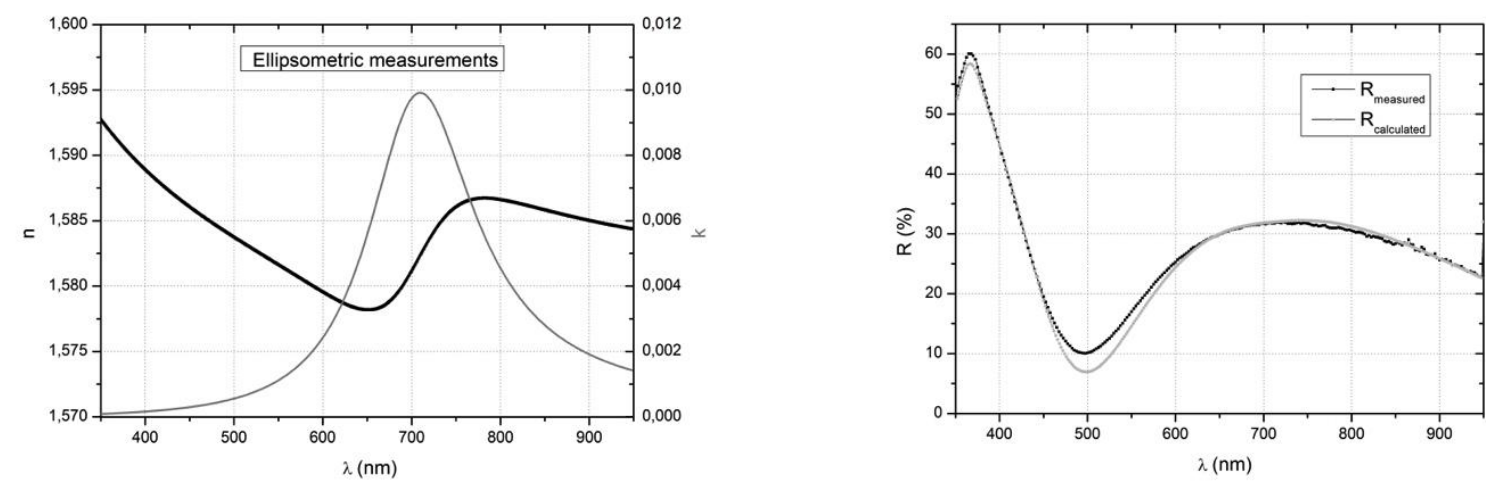

Figure 10. Left : Ellipsometric measurements on heterogeneous layer made of metallic nanoparticles embedded in PVP polymer. Right : Reflection spectrum measured and calculated by TMM thanks to ellipsometric measurements.

\subsection{Photonic crystals for light trapping in Organic Solar Cells}

In order to control the light inside a solar cell, some photonic structures can be added to the device. Here, we present the possibility to introduce photonic crystals inside an Organic Solar Cell (OSC), to improve the absorption of cell. This work is based on the modeling of the active layer under the shape of a photonic crystal slab. The structure modeled is presented on the figure 11, and consists of a stack of thin film layers, plus a double patterning of the active layer. Finite Difference Time Domain (FDTD) modeling have been used to calculate the photonic absorption spectra of two different structures, one planar and the other one with the photonic crystal slab. The figure 11 shows that the PC patterning boosts the absorption of the OSC, due to a Bloch mode coupling with a peak at $\lambda=704 \mathrm{~nm}$.

Several modeling at different angles were also done, showing that it is possible to obtain multiple Bloch resonances in the case of a large incidence cone. And finally, an absorption gain of around $15 \%$, with an $8^{\circ}$ incident angle cone, using a PC was obtained [5].

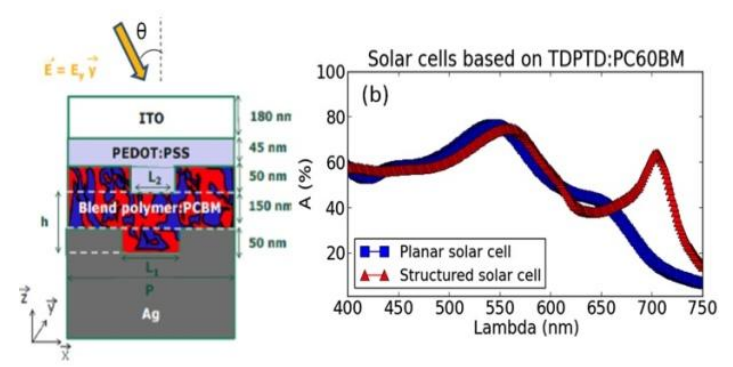

Figure 11. Left : Organic solar cell with a double photonic crystal patterning. Right : Absorption spectra for two different OSC structures (extracted from [5]).

\subsection{Color predicting in Organic Solar Cells}

The results presented in this part concern color and performance investigations of an organic solar cell, with PTB7:PC70BM as the active layer. The objective is to design solar cell with color specifications, still maintaining good performances, i.e. efficiency at the state of the art.

This study is based on a classical architecture of an OSC (see figure 12, on the left), which is a stack of thin film layers. The whole structure is optimized in terms of thickness of each layer, in order to control the interferential phenomena 
occurring in the multilayer stack. An ellipsometric study was done previously to obtain the optical indices of all the layers. First, we calculate the total number of photons absorbed inside the active layer under an AM1.5 illumination as functions of the $\mathrm{ZnO}$ layer thickness and of the active layer thickness. By taking into account the sensitivity of the human eye which is directly linked to the color perception process based on the CIE xyY.

Curves are drawn to present the different colors that can be obtained by varying the thicknesses of the $\mathrm{ZnO}$ layer and the PTB7:PC70BM layer. The different shapes (lines or curves) of the diagram correspond to different $\mathrm{ZnO}$ thicknesses (from 50 to $90 \mathrm{~nm}$, by $10 \mathrm{~nm}$ step). At the end of these modeling results, three solar cells ("Green G, Yellow Y, and Blue B) were experimentally realized with power conversion efficiencies of $6.73 \%, 7.10 \%$ and $7.59 \%$, respectively. The photograph images of the three solar cells, G, Y and B under different observation angles are presented near the chromaticity diagram. Finally, this work has shown the ability to control the color of the organic solar cells, while keeping a good efficiency.
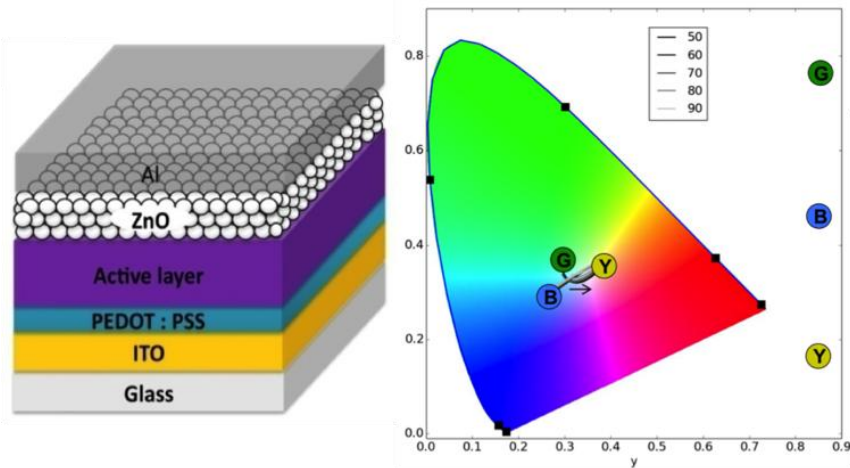

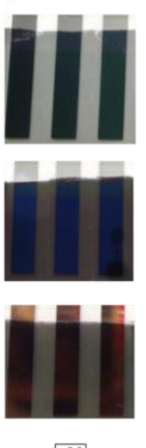

$8^{\circ}$

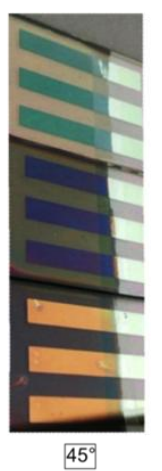

$45^{\circ}$

Figure 12. Left : Organic solar cell in a classical architecture. Right : Predicted color as a function of active layer and $\mathrm{ZnO}$ thicknesses (An arrow indicates the increase of the active layer thickness) and photograph images of the Green $(\mathrm{G})$, Blue (B) and Yellow (Y) solar cells at $8^{\circ}$ and $45^{\circ}$ (right) [21].

\section{CONCLUSION}

Nowadays, the materials used in optoelectronic devices are quite complex providing some difficulties to predict the optoelectronic behavior. In a first step, the knowledge of the optical properties of materials is necessary to design an optimized layer according specific properties.

Through this paper, we tried to present the optical behavior of thin film layers, studied through a set of tools, both experimental and modeling. We choose different examples of optical studies, mixing measurement and modeling, to show the complementarity of different set-up to obtain information on our structures. We also point out that these works can be performed on different layers, either organic or inorganic, on photonic structures or even on layers including metallic nanoparticles. This is possible thanks to both precise modeling and precise measurements.

Ellipsometry, spectrophotometry, PL, and PR characterizations are powerful tools to get some important parameters of a material. The combination of these techniques can also bring more information. Obviously, the use of other techniques, not described here, allows going more in details about the complete behavior of a thin film. However, this is not sufficient if we want to be confident in our measurements, and if we want to predict the optical behavior of our device. A complete modeling of the thin film layers, including the Physics of the material, allows concluding or predicting their performances. A better knowledge on the light management in the "new thin films layers" is now possible by combining accurate measurements and precise modeling. The efficiency of solar cells made of CIGS, perovskite, P3HT:ZnO, PC70BM can then be maximize; the color of solar cells can be designed; the optimal properties of PPV thin films containing metallic nanoparticles can be mastered. 


\section{REFERENCES}

[1] Locharoenrat K., [Optical Properties of Solids: An Introductory Textbook]. Pan Standford, Singapore, (2016).

[2] Flory F., Escoubas L., Le Rouzo J., Berginc G., Lee C., " Low-dimensional optics," J. Nanophoton. 0001, 9(1):093594. (2015).

[3] Le Rouzo J., Brückner J-B., Ferchichi A., Gourgon C., Berginc G., Escoubas L., " Optical properties of antireflective flat or rough patterned topped silicon cones gratings, " Advanced Device Materials, 1 (1), 23-26 (2015)

[4] Monestier F., Simon J-J., Torchio P., Escoubas L., Flory F., Bailly S., de Bettignies R., Guillerez S., Defranoux C., " Optical properties of antireflective flat or rough patterned topped silicon cones gratings, " Solar Energy Materials and Solar Cells, 91 (5), 405-410 (2007)

[5] Duché D., Masclaux C., Le Rouzo J., Gourgon C., " Photonic crystals for improving light absorption in organic solar cells," Journal of Applied Physics, 117 (5), 053108 (2015).

[6] Scholl J.A., Koh A.L., Dionne J.A., " Quantum plasmon resonances of individual metallic nanoparticles, "Nature, 483, 421-428 (2012).

[7] Vedraine S., Torchio P., Duché D., Flory F., Simon J-J., Le Rouzo J., Escoubas L., " Intrinsic absorption of plasmonic structures for organic solar cells, " Solar Energy Materials and Solar Cells 95, S57-S64 (2011).

[8] Green, M., [Third Generation Photovoltaics], Springer-Verlag Berlin Heidelberg Publishers, 1437-0379, XI, 160, (2003).

[9] Shockley W., Queisser H.J., " Detailed balance limit of efficiency of pn junction solar cells, " Journal of Applied Physics 32, 510 (1961).

[10] Hoppe H., Sariciftci N.S., " Organic solar cells: An overview, " Journal of Material Research 19 (7) (2004).

[11] Sessolo M., Bolink and H.J., " Perovskite solar cells join the major league " Science 350, 917 (2015).

[12] Bencheikh F., Duché D., Simon J-J., Escoubas L., " Ellipsometric study of the optical transitions of PC60BM and PC70BM thin films," Chemical Physics, 450-451, 102-108 (2015).

[13] Unold T., Gütay L., [Advanced Characterization Techniques for Thin Film Solar Cells], Wiley-VCH Ver lag GmbH \& Co. KGaA., Weinheim, Germany, D. Abou-Ras, T. Kirchartz, and U. Rau, Editors151-175 (2011).

[14] Ruiz C.M., Duché D., Le Rouzo J., [Experimental and Simulation Tools for Thin-Film Solar Cells], SPIE Press, Spotlight, in press (2016).

[15] Hamnett A., Gilman J., Batchelor R.A., " Theory of electroreflectance and photoreflectance of semiconductors," Electrochim. Acta , 37, (5) 949-956 (1992).

[16] Aspnes D.E., " Band nonparabolicities, broadening, and internal field distributions: the spectroscopy of FranzKeldysh oscillations," Physical Review B, 10 (10), 4228-4240 (1974).

[17] Ruiz C.M., Gonzalez-Pedro V., Moreau A., Le Rouzo J., Simon J-J., Mora-Sero I., Escoubas L., " Using combined photoreflectance and photoluminescence for understanding optical transitions in perovskites, " Proc. IEEE Photovoltaic Specialists Conf. (PVSC) (2015).

[18] Moreau A., Insignares-Cuello C., Escoubas L., Simon J-J., Bermudez V., Perez-Rodriguez A., Izquierdo-Roca, V., Ruiz C.M., " Impact of Cu-Au type domains in high current density CuInS2 solar cells," Solar Energy Materials and Solar Cells, 139, 101-107 (2015).

[19] Duché D., Bencheikh F., Ben Dkhil S., Gaceur M., Berton N., Margeat O., Ackermann J., Simon J-J., Escoubas L., " Optical performance and color investigations of hybrid solar cells based on P3HT:ZnO, PCPDTBT:ZnO, PTB7:ZnO and DTS(PTTh2)2:ZnO, " Solar Energy Materials and Solar Cells, 126, 197-204 (2014).

[20] Bencheikh F., Duché D., Ruiz C.M., Simon J-J., Escoubas L., " Study of Optical Properties and Molecular Aggregation of Conjugated Low Band Gap Copolymers: PTB7 and PTB7-Th," The Journal of Physical Chemistry C, 119 (43), 24643-24648 (2015).

[21] Ben Dkhil S., Duché D., Gaceur M., Thakur A.K., Bencheikh Aboura F., Escoubas L., Simon J-J., Guerrero A., Bisquert J., Garcia-Belmonte G., Bao Q., Fahlman M., Videlot-Ackermann C., Margeat O., Ackermann J., " Interplay of Optical, Morphological, and Electronic Effects of ZnO Optical Spacers in Highly Efficient Polymer Solar Cells," Advanced Energy Materials, 4 (18), 1400805 (2014). 